\title{
GRANITOS BORRACHUDOS: UM EXEMPLO DE GRANITOGÊNESE ANOROGÊNICA NA PORÇÃO CENTRAL DO ESTADO DE MINAS GERAIS
}

\author{
Maria Lourdes Souza Fernandes (*), Vitória Régia P.R.Oliveiros Marciano(*), \\ Robson Cota de Oliveira (*), José Marques Correia Neves (**) \& \\ Marcus Vinicius Diláscio (***)
}

\begin{abstract}
The Borrachudos Suite, formed by granitoid rocks that crops out in the central part of the Minas Gerais state, Brazil, presents very uniform chemical and mineralogical compositions.

Microscopic and $\mathrm{x}$-ray diffraction investigations show that these rocks are essencially constituted by microcline, albite, quartz, biotite, and hornblende. Allanite, zircon, magnetite, and ilmenite are the main accesory minerals, though xenotime, monazite, and epidote can be present. Biotite and hornblende are later crystallized.

They are alkaline rocks and plot as anorogenic granites on the geotectonic discrimination diagrams. The REE distribution pattern agrees with this classification. The geochemical data, to point a crustal origin The age about $1.7 \mathrm{Ga}$ obtained by Dossin et al. (1993) allows to link the Borrachudos granitoids to the opening of the Espinhaço Rift.
\end{abstract}

\section{INTRODUÇÃO}

A caracterização mineralógica, petrográfica e geoquímica de rochas da Suíte Borrachudos constituem o escopo deste trabalho.

Os Granitos Borrachudos, assim chamados por Dorr \& Barbosa (1963) afloram sob a forma de vários corpos na porção central do estado de Minas Gerais, a nordeste do Quadrilátero Ferrífero, distribuídos em ampla faixa na borda sudeste do Cráton do São Francisco (Fig. 1). A designação Suíte Borrachudos foi proposta por Grossi Sad et al. (1990).

Do ponto de vista do contexto geológico, as rochas mais antigas da região e que constituem o embasamento são gnaisses ortoderivados, aos quais Grossi Sad et al. (1990) denominaram Suíte Guanhães. Müller et al. (1986), baseando-se em dados petrográficos e da química dos elementos traços atribuem a estas rochas origem a partir de granito metamorfizado.

Sobre estas litologias repousam rochas supracrustais metavulcano-sedimentares, caracterizadas pela presença de xistos diversos com intercalações de anfibolitos, quartzitos, níveis ferruginosos e enriquecidos com manganês.

A este conjunto Grossi Sad et al. (1990) designaram Grupo Guanhães e corresponde a toda a área marcada em branco no mapa (fig. 1), onde os corpos da Suíte Guanhães não estão distinguidos.

Cortando as demais litologias estão os corpos da Suíte Borrachudos, uma rocha granítica, de granulometria média a grossa.
Estas rochas têm sido já objeto de investigações anteriores, como as de Dorr \& Barbosa (1963), Herz (1970) que as consideram pós a tardi-tectônicas com relação ao evento transamazônico; Schorscher \& Müller (1977), Schorscher (1992) que as interpretam como metassomatitos derivados de gnaisses e xistos do Embasamento; Chemale Jr. (1987) e Dussin (1994), atribuindo-lhes origem anorogênica e Grossi Sad et al. (1990) que propõem origem a partir dos ortognaisses da Suíte Guanhães em ambiente sin-colisional, de idade Minas.

Dossin et al. (1992) obtiveram idades U-Pb iguais a 1,7 Ga em zircão, pelo método de evaporação.

\section{ASPECTOS MACROSCÓPICOS E DE CAMPO}

As rochas da Suíte Borrachudos estão expostas naturalmente na forma de pães-de-açúcar ou no leito dos rios. Exposições artificiais são fornecidas por pedreiras e cortes de estradas. A área de exposição é descontínua e, devido ao espesso manto regolítico que as recobre, os afloramentos podem ser escassos.

Tratam-se de rochas bastante homogêneas, de granulação grosseira a média, de cor cinza a rosa quando fresca, a cinza amarelada, quando intemperizada. A biotita é o principal mineral máfico, seguida pelo anfibólio. Ambos aparecem como marcadores do fluxo magmático. A foliação magmática confere aspecto orientado a estes granitos, caracterizado pelo arranjo dos minerais máficos em níveis descontínuos de quatro a cinco centímetros de 


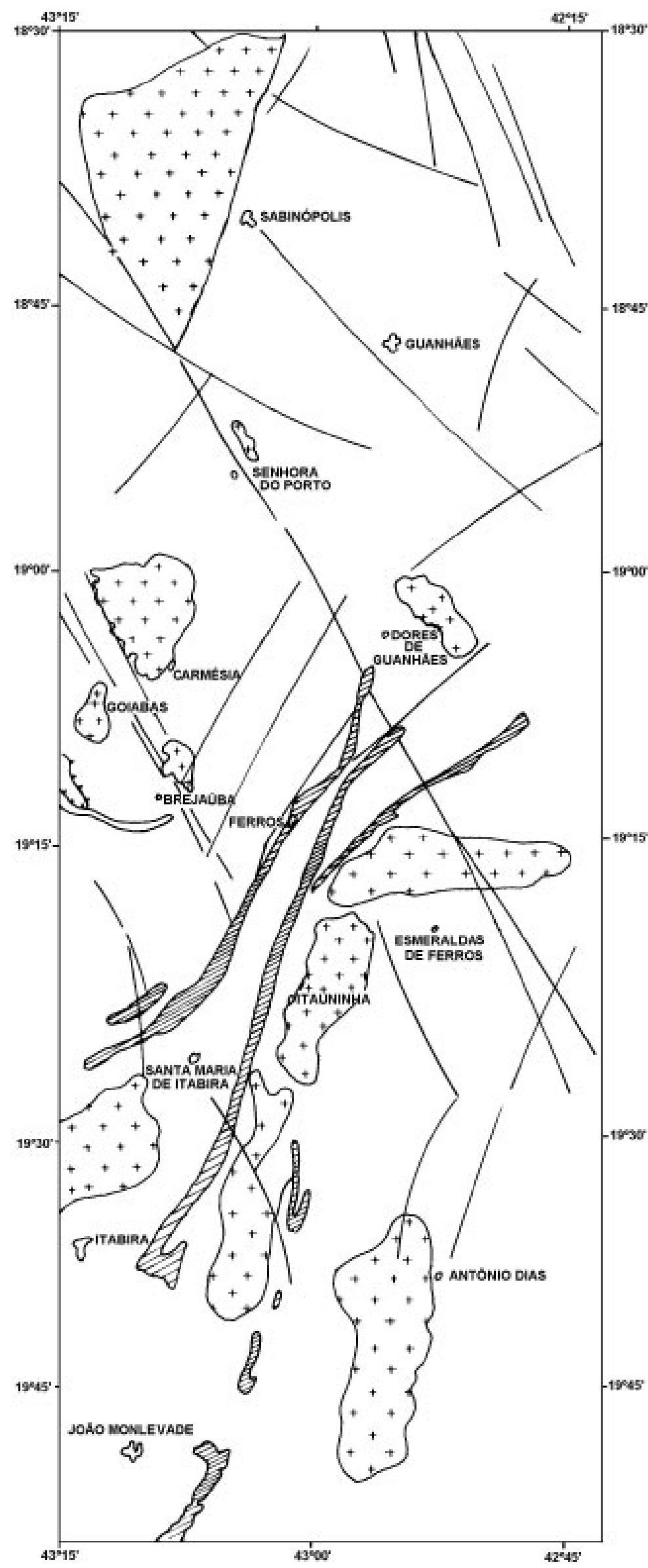

Figura 1: Mapa de Localização.

Figure 1: Localization Map.
MAPA DE LOCALIZAÇÃO

DAS AMOSTRAS

ESTUDADAS

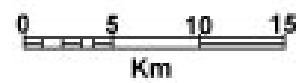

CIDADE
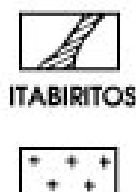

GRANITO BORRACHUDOS

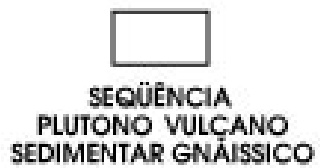

PROJETO MAPAS METALOGENÉtICOS E DE PREVISȦO DE RECURSOS MINERANS

CARTAS METALOGENÉTICAS DE:

IPATINGA - 5E.23-2-D

GUANHĀES - SE.23-2-B

ESCALA 1250000

DNPM/CPRM (MODIFCADO)

LOCAUZAÇÃO DA ÁREA DE DE PESGUISA NO ESTADO DE MINAS GERAIS

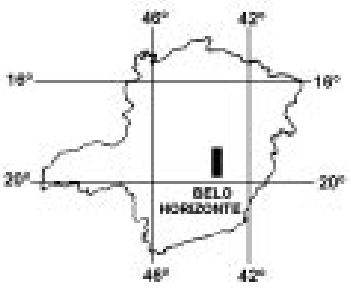


comprimento. Nas porções mais internas estas feições desaparecem. Tais observações são particularmente válidas para o corpo aflorante na região de Dores de Guanhães.

Nesta região, onde trabalhos de mapeamento litofaciológico estão em andamento, é possível, segundo Carneiro \& Romeiro (1994) apontar a presença de 3 fácies, com base no mineral máfico presente: anfibóliogranito, biotita-anfibólio-granito e biotita-granito, esta última de maior expressão areal.

\section{ESTUDOS POR DIFRATOMETRIA DE RAIOS-X}

A fim de melhor caracterizar a composição mineralógica das rochas da Suíte, 8 amostras foram analisadas por Difratometria de Raios-X. Para tanto, cada amostra foi moída e submetida à separação densimétrica via bromofórmio. A fração leve foi separada e reservada. A fração pesada foi passada no imã e, o material restante, submetido à diferentes amperagens no separador magnético Franz, gerando um total de 8 alíquotas por amostra (incluindo a fração leve). Cada uma das alíquotas foi analisada por difratometria de raios-X. Os resultados obtidos podem ser observados na tabela 1 .

\begin{tabular}{|c|c|c|c|c|c|c|}
\hline & 4540 & 5086 & 5088 & 5218 & 5220 & 5227 \\
\hline$d<2,85$ & Mi-Ab-Qz & Mi-Ab-Qz & Mi-Ab-Qz & Mi-Ab-Qz & Mi-Ab-Qz & Mi-Ab-Qz \\
\hline$d=2,85$ & & & Mo & Mi-Ab-Qz & Mi-Ab-Oz & \\
\hline $\begin{array}{c}\mathrm{d}=2,85 \\
\text { Imã }\end{array}$ & $\begin{array}{l}\mathrm{Mt}-\| \\
\mathrm{He}-\mathrm{Ho}\end{array}$ & M & iMt & Mt-Bi & $\mathrm{Mt}-\|-\mathrm{Bi}$ & Mt-II-Bi \\
\hline $\begin{array}{c}d>2,85 \\
1 A\end{array}$ & $\begin{array}{l}\text { Bi-Mo- } \\
\text { II-Ho }\end{array}$ & & $\begin{array}{l}\text { Zr-Al- } \\
\text { Ho-Xe }\end{array}$ & $\begin{array}{l}\text { Bi-Ho- } \\
\text { Al-Ca }\end{array}$ & Bi-Al-Ho & Bi-Ho-II \\
\hline $\begin{array}{c}d>2,85 \\
2 A\end{array}$ & Bi-Ho & $\begin{array}{l}\text { Bi-Ho- } \\
\text { Al-Mz- } \\
\text { Zr-II }\end{array}$ & $\begin{array}{l}\text { Bi-Ho- } \\
\text { II-Ca }\end{array}$ & Bi-Al-Ca & Bi-Ho-Zr & Ho-II \\
\hline $\begin{array}{c}d>2,85 \\
3 A\end{array}$ & $\begin{array}{l}\text { Bi-Mo- } \\
\text { II-Ho- } \\
\text { Mz-Z }\end{array}$ & $\begin{array}{l}\text { Bi-Ho- } \\
\text { Zr-Mz-II }\end{array}$ & & & & \\
\hline $\begin{aligned} d & >2,85 \\
& >3 \mathrm{~A}\end{aligned}$ & $\begin{array}{c}\text { Mo-Tr- } \\
\text { Zr-Mz- } \\
\text { Ep-Al }\end{array}$ & $\begin{array}{l}\text { Mi-Ab- } \\
\text { Qz-Xe- } \\
\text { Ti-Mo }\end{array}$ & & & & \\
\hline
\end{tabular}

Tabela 1: Composição mineralógica dos Granitos Borrachudos, segundo dados de difratometria de raios- $X$.

Table 1: Mineralogical composition of the Borrachudos Suite, after diffractometric data. $M i=$ microclina, $A b=$ albita,$Q z=$ quartzo,

$M t=$ magnetita,$I l=$ ilmenita, $\mathrm{He}=$ hematita,

$H o=$ hornblenda, $M z=$ monazita, $\mathrm{Zr}=$ zircão,

$E p=$ epidoto, $A l=$ allanita,$X e=x e n o t i m a$ Ca=caulinita

É importante ressaltar que a presença de minerais da fração leve em porções de mais alta amperagem se deve ao fato de a moagem não ter conseguido desagregar completamente os minerais.

Observa-se ainda que as amostras do corpo de Dores de Guanhães (5218 - 5219 - 5220 - 5227) têm mineralogia mais simples que as demais, revelandose compostas essencialmente por microclina, albita, quartzo, biotita , além de hornblenda, allanita e zircão. Os principais minerais opacos são magnetita e ilmenita. Muscovita e caulinita são encontradas esporadicamente.

Fluorita e apatita são minerais acessórios observados microscopicamente e cujas presenças não foram acusadas pela difratometria.

Além dos minerais retrocitados, a difratometria apontou a presença de minerais como xenotima, monazita, titanita e epidoto em amostras dos outros corpos, que não o da região de Dores de Guanhães

\section{ASPECTOS MICROSCÓPICOS}

Os estudos petrográficos realizados em lâminas delgadas confirmam os resultados obtidos por difratometria de raios- $x$, as exceções já tendo sido citadas.

Não obstante as variações faciológicas observadas na região de Dores de Guanhães, cujo significado e extensão não é possível ainda compreender; as relações texturais das rochas desta suíte são bastante monótonas. Dentre as lâminas observadas, apenas aquelas do corpo de Itabira contrariam esta regra

As rochas da Suíte Borrachudos têm como constituintes essenciais a microclina mesopertítica, quartzo e, mais raramente plagioclásio, além de biotita e/ou hornblenda. Os principais minerais acessórios são zircão (em cristais relativamente grandes), allanita, apatita e fluorita. Os minerais opacos são magnetita e ilmenita, em complexas relações de exsolução, como revelado pela microscopia eletrônica, além de pirita.

De um modo geral, os granitos Borrachudos apresentam textura granular. As relações entre os cristais, como as inclusões de feldspato na biotita e anfibólio, a forma anédrica destes dois últimos e suas presenças como marcadores do fluxo magmático permitem apontar uma seqüência de cristalização invertida relativamente à série de Bowen, caracterizada pela cristalização precoce do feldspato e tardia dos minerais máficos. Tal feição tem sido observada em granitos alcalinos de outras partes do mundo (Whalen et al. 1987) e também obtidas em resultados experimentais (Clemens et al. 1986).

As lâminas do corpo de Itabira apresentam intensa transformação textural e mineral. A textura é granoblástica, estando os feldspatos intensamente caulinizados. Diferentemente dos outros corpos, o principal mineral máfico, a biotita, apresenta coloração verde.

\section{GEOQUÍMICA}

Oito amostras destes granitos foram analisadas quanto aos elementos maiores, menores, elementos terras raras e alguns traços. Destas, quatro foram coletadas do corpo aflorante na região de Dores de Guanhães e as demais são de Itauninha, Goiabas, Sabinópolis e Itabira (fig. 1). Os resultados obtidos e a composição normativa destas rochas estão contidos nas tabelas 2 e 3 , respectivamente. 


\begin{tabular}{|c|c|c|c|c|c|c|c|c|}
\hline & 4540 & 5077 & 5086 & 5088 & 5218 & 5219 & 5220 & 5227 \\
\hline $\mathrm{SiO}_{2}$ & 76,3 & 76,2 & 76,1 & 73,4 & 71 & 77,5 & 74,7 & 75,6 \\
\hline $\mathrm{Al}_{2} \mathrm{O}_{3}$ & 10,62 & 10,81 & 11,04 & 12,13 & 13,72 & 10,98 & 11,43 & 11,05 \\
\hline $\mathrm{TiO}_{2}$ & 0,13 & 0,26 & 0,23 & 0,19 & 0,1 & 0,1 & 0,2 & 0,19 \\
\hline $\mathrm{Fe}_{2} \mathrm{O}_{3}$ & 0,12 & 0,59 & 0,56 & 0,92 & 1,24 & 0,06 & 0,59 & 0,4 \\
\hline $\mathrm{FeO}$ & 1,55 & 2,18 & 1,57 & 1,44 & 1,15 & 1,5 & 1,51 & 1,71 \\
\hline $\mathrm{CaO}$ & 0,85 & 0,7 & 0,77 & 0,6 & 0,61 & 0,65 & 0,87 & 0,65 \\
\hline $\mathrm{MgO}$ & 0,02 & 0,02 & 0,03 & 0,09 & 0,01 & 0,01 & 0,09 & 0,04 \\
\hline $\mathrm{MnO}$ & 0,01 & 0,03 & 0,02 & 0,03 & 0,02 & 0,01 & 0,02 & 0,03 \\
\hline $\mathrm{Na}_{2} \mathrm{O}$ & 3,88 & 5,03 & 4,38 & 4,99 & 5,19 & 4,65 & 4,58 & 3,78 \\
\hline $\mathrm{K}_{2} \mathrm{O}$ & 5,48 & 5,3 & 5,48 & 5,25 & 5,9 & 5,6 & 5,25 & 5,3 \\
\hline $\mathrm{H}_{2} \mathrm{O}-$ & 0,07 & 0,07 & 0,07 & 0,13 & 0,19 & 0,06 & 0,18 & 0,12 \\
\hline $\mathrm{H}_{2} \mathrm{O}+$ & 0,51 & 0,43 & 0,26 & 0,25 & 0,35 & 0,42 & 0,19 & 0,13 \\
\hline $\mathrm{P}_{2} \mathrm{O}_{5}$ & 0,01 & 0,02 & 0,02 & 0,01 & 0,01 & 0,01 & 0,03 & 0,01 \\
\hline $\mathrm{Li}$ & 510 & 480 & 460 & 455 & 555 & 520 & 495 & 325 \\
\hline Th & 72 & 50 & 45 & 40 & 119 & 86 & 49 & 34 \\
\hline $\mathrm{Ba}$ & 131 & 272 & 705 & 499 & 67 & 75 & 471 & 366 \\
\hline Ta & $<5$ & 7 & $<5$ & $<5$ & 20 & $<5$ & $<5$ & $<5$ \\
\hline $\mathrm{Nb}$ & 39 & 56 & 34 & 51 & 183 & 58 & 15 & 24 \\
\hline Cs & $<5$ & $<5$ & $<5$ & $<5$ & 9 & $<5$ & $<5$ & $<5$ \\
\hline U & 15 & $<10$ & $<10$ & $<10$ & 33 & $<10$ & $<10$ & $<10$ \\
\hline $\mathrm{Rb}$ & 512 & 217 & 209 & 254 & 529 & 456 & 233 & 210 \\
\hline $\mathrm{HF}$ & $<8$ & 16 & $<8$ & 9 & $<8$ & $<8$ & $<8$ & $<8$ \\
\hline $\mathrm{Sr}$ & 34 & 27 & 5 & 38 & 15 & 15 & 94 & 48 \\
\hline Y & 212 & 137 & 109 & 105 & -12 & 211 & 75 & 94 \\
\hline $\mathrm{Zr}$ & 314 & 621 & 381 & 426 & 137 & 262 & 297 & 324 \\
\hline V & $<5$ & $<5$ & $<5$ & $<5$ & $<5$ & $<5$ & $<5$ & $<5$ \\
\hline $\mathrm{Cu}$ & 44 & 2,5 & 3,7 & 2,9 & 2,8 & 10 & 8,5 & 1,4 \\
\hline $\mathrm{Zn}$ & 65 & 102 & 85 & 90 & 40 & 37 & 39 & 97 \\
\hline $\mathrm{Be}$ & 11,3 & 4,4 & 4,1 & 7,1 & 7,6 & 2,8 & 5,1 & 3,4 \\
\hline $\mathrm{Pb}$ & 100 & 78 & 79 & 72 & 109 & 83 & 71 & 73 \\
\hline $\mathrm{Zr}$ & 264 & 73 & 132 & 114 & 108 & 216 & 197 & 156 \\
\hline $\mathrm{Cr}$ & $<2$ & $<2$ & $<2$ & $<2$ & $<2$ & $<2$ & $<2$ & $<2$ \\
\hline Ga & 35,5 & 24,9 & 31,1 & 34,8 & 39,5 & 27,7 & 23,4 & 23,6 \\
\hline La & 145,6 & 234,6 & 137,6 & 130,5 & 77,3 & 79,9 & 95,3 & 124,7 \\
\hline $\mathrm{Ce}$ & 259,1 & 438,6 & 251,2 & 242,4 & $\mid 145,4$ & 159,2 & 180,5 & 234,8 \\
\hline $\mathrm{Nd}$ & 93 & 178,1 & 102,1 & 100,6 & 52,5 & 62,1 & 68,6 & 95 \\
\hline Sm & 17,76 & 30,32 & 17,97 & 19,09 & 10,99 & 13,47 & 11,71 & 17,41 \\
\hline $\mathrm{Eu}$ & 0,5 & 1,13 & 1,54 & 1,41 & 0,32 & 0,5 & 0,88 & 0,87 \\
\hline Gd & 17,41 & 24,73 & 15,82 & 17,71 & $\mid$\begin{tabular}{|l|l} 
& 11,07
\end{tabular} & 14,4 & 9,61 & 15,41 \\
\hline Dy & 19,7 & 20,25 & 13,44 & 14,75 & 12,99 & 17,52 & 8,22 & 13,26 \\
\hline Ho & 4,45 & 4,25 & 2,83 & 2,98 & 2,94 & 4,08 & 1,75 & 2,82 \\
\hline Er & 13,54 & 11,25 & 7,59 & 7,73 & 8,63 & 12,45 & 4,58 & 7,29 \\
\hline$Y b$ & 13,81 & 10 & 6,91 & 6,27 & 9 & 12,91 & 4,1 & 6,2 \\
\hline Lu & 1,59 & 1,19 & 0,82 & 0,7 & 1,02 & 1,53 & 0,47 & 0,73 \\
\hline Y & 127 & 103 & 70 & 72 & 85 & 120 & 48 & 68 \\
\hline
\end{tabular}

Tabela 2: Composição química dos Granitos Borrachudos.

Table 2: Chemical composition of Borrachudos Granitoids.

\begin{tabular}{|c|c|c|c|c|c|c|c|c|}
\hline & $\mathbf{4 5 4 0}$ & $\mathbf{5 0 7 7}$ & $\mathbf{5 0 8 6}$ & $\mathbf{5 0 8 8}$ & $\mathbf{5 2 1 8}$ & $\mathbf{5 2 1 9}$ & $\mathbf{5 2 2 0}$ & $\mathbf{5 2 2 7}$ \\
\hline $\mathrm{Qz}$ & 36,65 & 33,16 & 33,03 & 26,71 & 19,02 & 35,3 & 30,57 & 33,58 \\
\hline $\mathrm{Zr}$ & 0,06 & 0,12 & 0,08 & 0,09 & 0,03 & 0,05 & 0,06 & 0,07 \\
\hline $\mathrm{Or}$ & 32,55 & 31,41 & 32,47 & 31,13 & 35,07 & 33,27 & 31,12 & 31,41 \\
\hline $\mathrm{Ab}$ & 22,06 & 24,22 & 24,48 & 31,37 & 35,47 & 23,2 & 27,62 & 26,04 \\
\hline $\mathrm{Ac}$ & 0,35 & 1,71 & 1,62 & 2,66 & 3,59 & 0,17 & 1,71 & 1,16 \\
\hline $\mathrm{Met} \mathrm{Na}$ & 2,41 & 3,82 & 2,5 & 1,82 & 1,02 & 3,71 & 2,14 & 1,08 \\
\hline $\mathrm{Di}$ & 3,74 & 3,05 & 3,38 & 2,7 & 2,66 & 2,84 & 3,77 & 2,91 \\
\hline $\mathrm{Hi}$ & 0,72 & 2,07 & 1,39 & 1,21 & 0,6 & 1,13 & 0,74 & 1,46 \\
\hline $\mathrm{II}$ & 0,25 & 0,49 & 0,44 & 0,36 & 0,19 & 0,19 & 0,38 & 0,36 \\
\hline $\mathrm{Ap}$ & 0,02 & 0,05 & 0,05 & 0,02 & 0,02 & 0,02 & 0,07 & 0,02 \\
\hline $\mathrm{Ep}$ & 1,37 & 1,29 & 1,23 & 1,22 & 1,49 & 1,39 & 1,33 & 0,87 \\
\hline
\end{tabular}

Tabela 3 - Composição normativa dos Granitos

Borrachudos.

Table 3 - Normative composition of Borrachudos Granitoids.

$Q z=$ quartzo, $\mathrm{Zr}=$ zircão, $\mathrm{Or}=$ ortoclásio, $A b=$ albita, Ac = acmita, Met.Na = metassilicato de sódio, $\mathrm{Di}=$ diopsídio, hiperstênio,Il = ilmenita, ap = apatita, Ep = epidoto.

Os elementos maiores $\left(\mathrm{SiO}_{2}, \mathrm{Al}_{2} \mathrm{O}_{3}, \mathrm{Fe}_{2} \mathrm{O}_{3}, \mathrm{CaO}\right.$, $\mathrm{MgO}$ e $\mathrm{TiO}_{2}$ ) e alguns traços ( $\mathrm{Ba}, \mathrm{Sr}, \mathrm{Cr}, \mathrm{V}, \mathrm{Cu}, \mathrm{Zn}$, $\mathrm{Be}, \mathrm{Pb}$ e $\mathrm{Ga}$ ) foram determinados por ICP. $\mathrm{Na}_{2} \mathrm{O}, \mathrm{K}_{2} \mathrm{O}$ e $\mathrm{Li}_{2} \mathrm{O}$ foram medidos por fotometria de chama; $\mathrm{FeO}$, por volumetria; $\mathrm{P}_{2} \mathrm{O}_{5}$ por colorimetria; $\mathrm{H}_{2} \mathrm{O}^{+}$e $\mathrm{H}_{2} \mathrm{O}^{-}$, por gravimetria. Os elementos traços $\mathrm{Th}, \mathrm{Ta}, \mathrm{Nb}, \mathrm{Cs}$, $\mathrm{U}, \mathrm{Rb}, \mathrm{Hf}$ e $\mathrm{Zr}$ foram analisados por fluorescência de raios- $\mathrm{x}$. Os elementos terras raras e o $\mathrm{Y}$ foram determinados por ICP, utilizando-se monocromador específico para os ETR.

As rochas da Suíte Borrachudos caracterizam-se por marcada uniformidade composicional, o que pode ser visualizada pela concentração dos pontos nos diagramas geoquímicos.

Estas rochas caracterizam-se pelo elevado teor em sílica $\left(\mathrm{SiO}_{2}>71,0 \%\right), \mathrm{Na}_{2} \mathrm{O}+\mathrm{K}_{2} \mathrm{O}>\mathrm{Al}_{2} \mathrm{O}_{3}$, baixíssimos teores de $\mathrm{MgO}(<0.09 \%)$, presença de acmita e hiperstênio normativos.

A figura 2 apresenta o diagrama Q-A-P, de Le Maitre (1989), construído a partir da composição normativa dos Granitos Borrachudos. A maior parte das amostras recai no campo dos sieno-granitos.

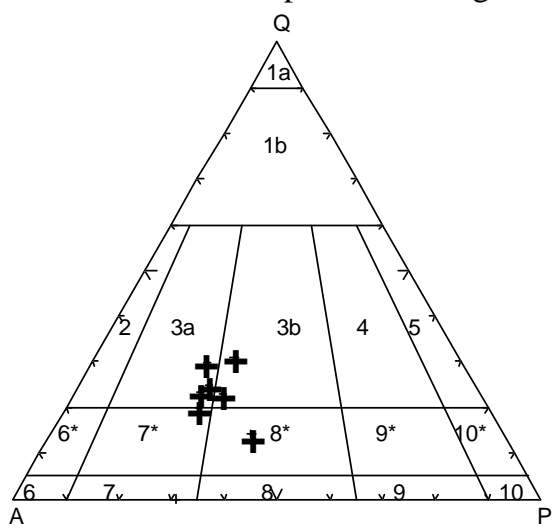

Fig 2 - Diagrama Q-A-P, de Le Maitre (1989).

Fig.2 - Q-A-P diagram, after Le Maitre (1989). 
Quando representadas no diagrama de alcalinidade de Wright (1969) - figura 3 - as litologias da Suíte Borrachudos projetam-se no campo das rochas peralcalinas, o que é confirmado pelo índice de Shand apresentado na figura 4.

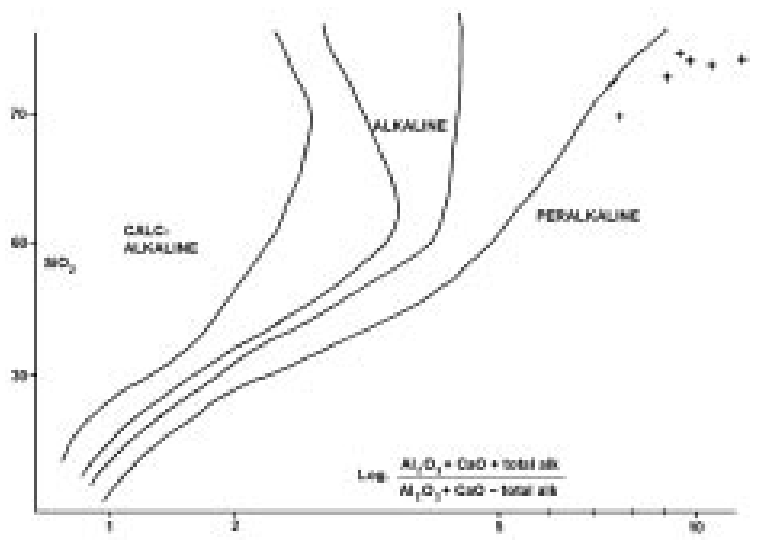

Figura 3:Índice de Alcalinidade de Wright 1969.

Figure 3: Alkalinity Index, after Wright 1969.

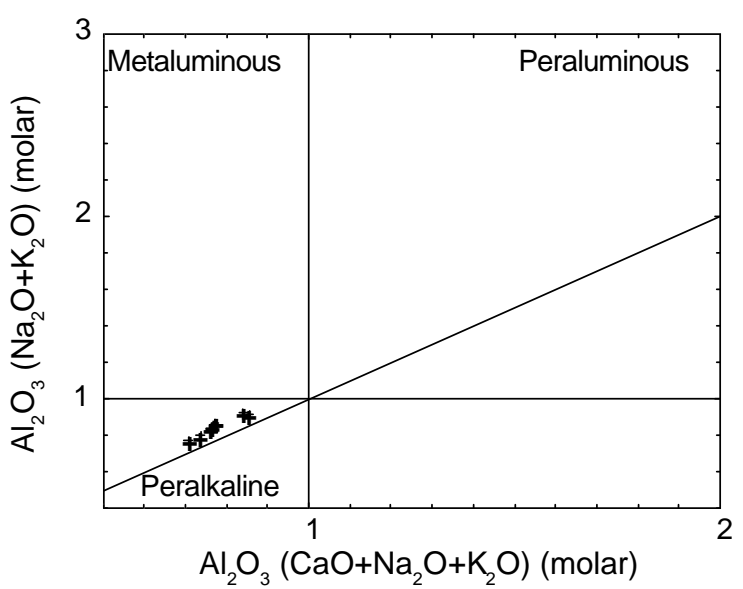

Figura 4: Índice de Alcalinidade de Shand.

Figure 4: Alkalinity Index, after Shand.

Os diagramas $\mathrm{Al}_{2} \mathrm{O}_{3}$ x $\mathrm{SiO}_{2}$ e $\mathrm{FeO} * / \mathrm{FeO}^{*}+\mathrm{MgO}$ x $\mathrm{SiO}_{2}$, de Maniar \& Piccoli (1989), mostrados nas figuras 5 e 6 respectivamente, são discriminativos para as rochas estudadas, que plotam nitidamente nos campos dos granitos ligados a rifteamento.

A observação da figura 7, que traz o diagrama $R_{1} x$ $\mathrm{R}_{2}$, de La Roche et al. (1980), com as subdivisões de Batchelor \& Bowden (1985), revela que os pontos recaem no campo 5, isto é, o campo dos granitos anorogênicos.

Diagramas envolvendo elementos traços para caracterização geotectônica de rochas granitóides foram propostos por Pearce et al. (1984). Tanto na figura 8: diagrama $\log \mathrm{Y}+\mathrm{Nb}$ x $\log \mathrm{Rb}$, como na figura 9: diagrama $\log \mathrm{Y} \times \log \mathrm{Nb}$, as amostras da Suíte Borrachudos projetam-se no campo dos granitos intraplacas.

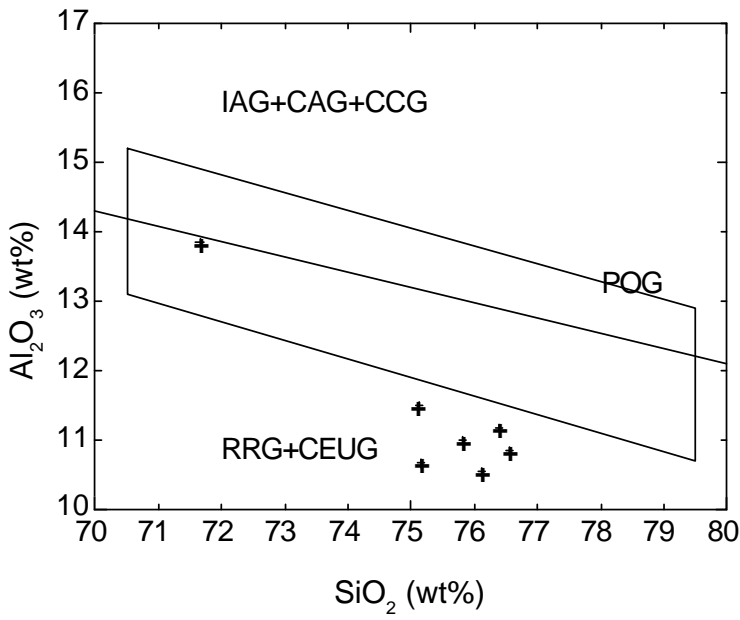

Figura 5: Diagrama $\mathrm{Al}_{2} \mathrm{O}_{3} \times \mathrm{SiO}_{2}$, de Maniar \& Piccoli 1989.

Figure 5: $\mathrm{Al}_{2} \mathrm{O}_{3} \times \mathrm{SiO}_{2}$ diagram, after Maniar \& Piccoli 1989.

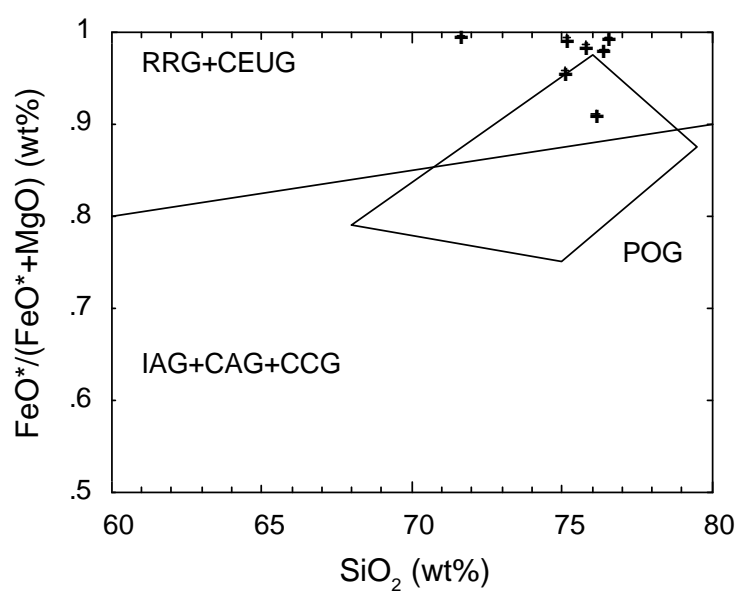

Figura 6: Diagrama $\mathrm{FeO} * / \mathrm{FeO} *+\mathrm{MgO} \times \mathrm{SiO}_{2}$, de Maniar \& Piccoli 1989.

Figure 6: $\mathrm{FeO} * / \mathrm{FeO}^{*}+\mathrm{MgO} \times \mathrm{SiO}_{2}$ diagram, after Maniar \& Piccoli 1989.

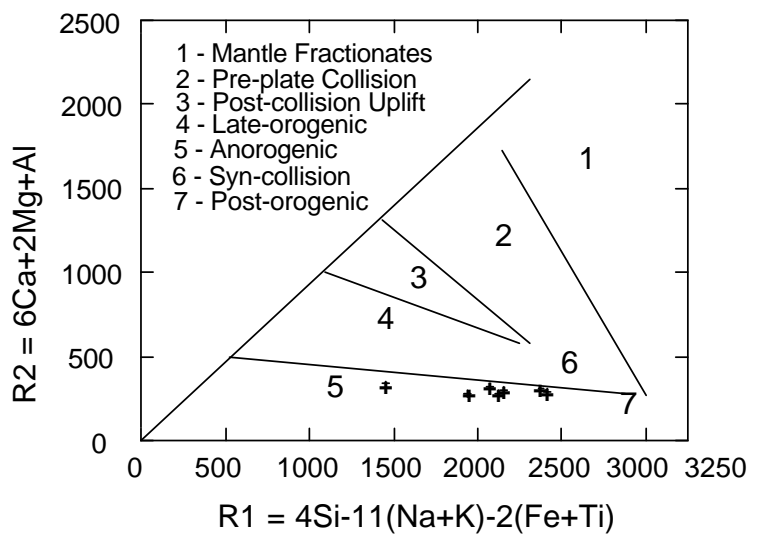

Figura 7: Diagrama $R_{1} \times R_{2}$, de La Roche et al. (1980), com as subdivisões de Batchelor \& Bowden (1985).

Figure 7: $R_{1} \times R_{2}$ diagram, after La Roche et al. (1980), with the modifications of Batchelor \& Bowden (1985). 


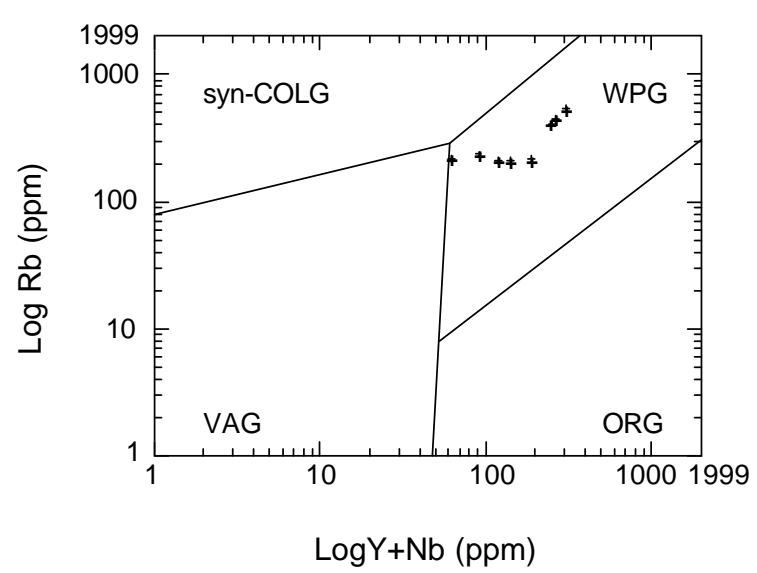

Figura 8 - Diagrama, de Pearce et al. 1984. Figure $8-\log Y+N b \times \log$ Rb diagram after Pearce et al. 1984.

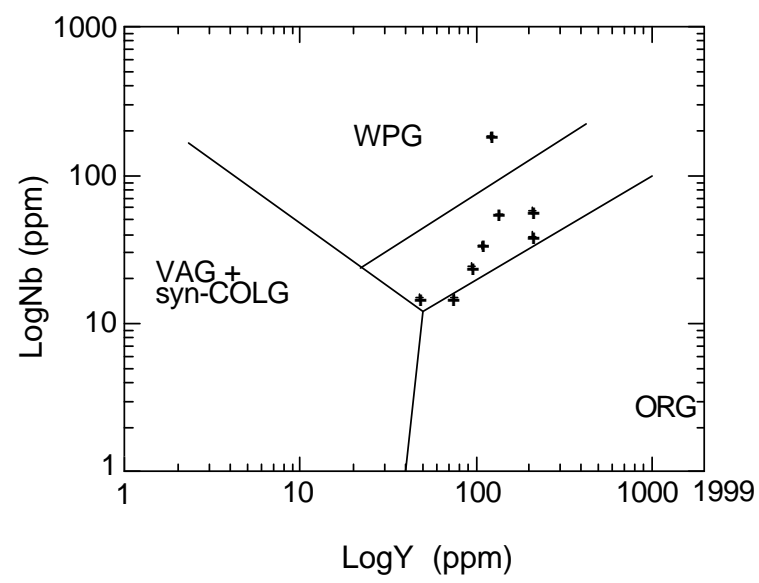

Figura 9: Diagrama $\log Y \times \log N b$, de Pearce et al. 1984.

Figure 9: $\log Y \times \log N b$ diagram, after Pearce et al. 1984.

O aracnograma da figura 10 mostra a distribuição de alguns dos elementos incompatíveis normalizados pelos valores do granito da cadeia oceânica (ORG), conforme proposto por Pearce et al. (1984). O padrão apresentado é análogo ao dos granitos intra-placas daqueles autores, como caracterizado pelo enriquecimento em $\mathrm{Rb}$ e Th relativamente ao $\mathrm{Nb}$ e $\mathrm{Ta}$ e anomalia positiva em $\mathrm{Ce}$ e $\mathrm{Sm}$ relativamente aos seus vizinhos. Tais enriquecimentos seletivos podem ser atribuídos ao envolvimento crustal na origem destas rochas.

Os granitos Borrachudos caracterizam-se por apresentar elevado conteúdo em ETR, sendo a média dos ETR igual a 530,45. As concentrações em ETR foram normalizadas pela do condrito, segundo valores de Evensen (1978) e os resultados, utilizados na construção de curvas de distribuição, mostradas na figura 11.

Observa-se forte uniformidade no padrão de distribuição dos ETR, exceção feita à amostra proveniente da região de Itabira, cuja mineralogia e

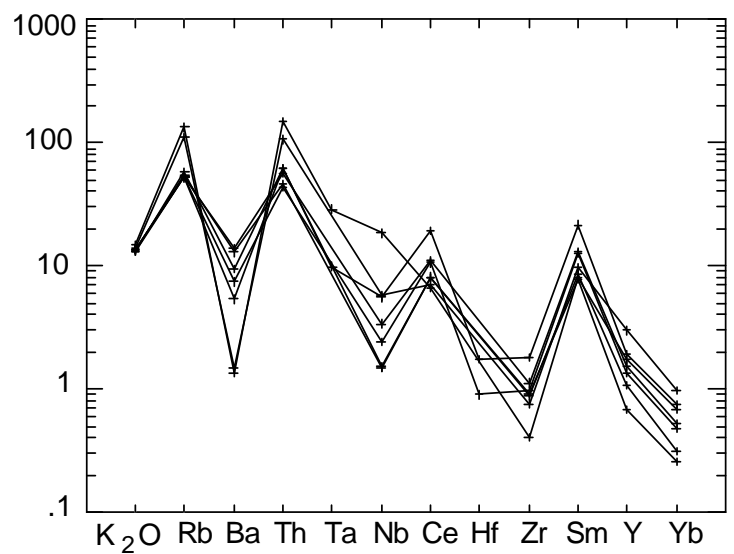

Figura 10: Aracnograma de Pearce et al. - 1984. Figure 10: Spidergram, after Pearce et al. - 1984.

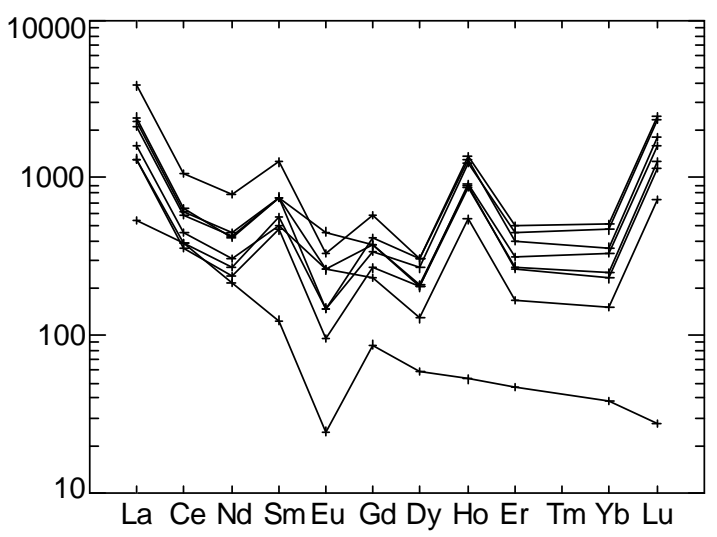

Figura 11: Distribuição dos ETR, normalizada pelo condrito, para rochas da Suíte Borrachudos.

Figure 11: Chondrite-normalized REE-patterns for Borrachudos Granitoids.

textura são também discordantes das demais amostras.

A feição mais marcante é a presença de acentuada anomalia negativa em Eu (em torno de 0,04), enriquecimento em ETRL relativamente aos ETRP $\left[(\mathrm{La} / \mathrm{Yb})_{\mathrm{N}}=1,08\right]$, ausência de fracionamento dos ETRP. Tal padrão de distribuição dos ETR é compatível com a química dos elementos maiores e traços destas rochas, sendo característico dos granitos alcalinos (Zhonggang 1982; Collins et al. 1982).

Segundo Bowden et al. (1974), é possível considerar que a depleção em Eu, acoplada ao enriquecimento em ETR médios está relacionada à precipitação precoce do feldspato pertítico e à cristalização tardia do anfibólio alcalino, a partir de líquidos de baixa temperatura enriquecidos em voláteis.

\section{CONCLUSÕES}

Os Granitos Borrachudos apresentam características como:

- sua composição mineralógica e sequência de cristalização, caracterizada pela cristalização tardia da biotita e anfibólio;

- índice de alcalinidade; 
- projeção dos dados nos diagramas de discriminação geotectônica;

- padrão de distribuição dos ETR, marcado por acentuada anomalia negativa em Eu e ausência de fracionamento em ETRP relativamente aos condritos; que levam à sua classificação inequívoca como granitos do tipo A, isto é, alcalinos e anorogênicos

O padrão de distribuição dos elementos incompatíveis no aracnograma de Pearce et al. (1984) são indicativos de sua origem a partir de rochas crustais, o que está de acordo com os resultados obtidos por Dussin (1994) através de estudos isotópicos.

As idades em torno de $1.7 \mathrm{Ga}$ obtidas por Dossin et al. (1993) permitem relacioná-los à abertura do rift Espinhaço.

\section{AGRADECIMENTOS}

Os autores agradecem o apoio financeiro da FAPEMIG e PRPq/UFMG.

\section{REFERÊNCIAS BIBLIOGRÁFICAS}

BATCHELOR, R.A. \& BOWDEN,P.1985. Petrogenetic interpretation of granitoid rock series using multicationic parameters. Chem. Geol., 48: 45-55.

BOWDEN,P. \& WHITLEY,J.E. 1974. Rare-earth patterns in alkaline and associated granites. Lithos,7: 15-21.

CARNEIRO,F.A. \& ROMEIRO,J.C.P. 1994 . Mapeamento Geológico de um corpo granitóide do tipo Borrachudos, na região de Dores de Guanhães, MG. Trabalho de Graduação, UFMG, 33 p. Ined.

CLEMENS JR.,F. 1987. Gênese das rochas graníticas do tipo Borrachudos. In: CONGRESSO BRASILEIRO DE GEOQUÍMICA, 1. Anais... p.171-186.

CLEMENS,J.D.; HOLLOWAY,J.R. \& WHITE, A.J.R. 1986 . Origin of an A-type granite: Experimental constraints. American Mineralogist, 71:317-324.

COLLINS, W.J.; BEAMS, S.D.; WHITE, A.J.R. \& CHAPPEL, B.W. 1982. Nature and origin of A-type granites with particular reference to Southeastern Australia. Contr. Mineral Petrol., 80:189-200

DORR,J.V.N. \& BARBOSA,A.L.M. 1963 . Geology and ore deposits of the Itabira District, Minas Gerais, Brazil. US Geol. Surv. Prof. Pap. 341-C, 110 p. Washington.

DOSSIN,I.A.; DOSSIN,T.M.; CHARVET,J.; COCHERIE,A. \& ROSSI,P. 1993 . Single-zircon dating by step-wise Pbevaporation of Middle Proterozoic Magmatismo in the Espinhaço Range, Southeastern São Francisco Craton (Minas
Gerais, Brazil). In: SIMPÓSIO DO CRÁTON DO SÃO FRANCISCO, 2. Salvador, 1993. Anais... 39-42.

DUSSIN,T.M. 1994 . The Borrachudos Suite, Mesoproterozoic Atype Granitic Magmatism in the Southeastern São Francisco Craton (SE Brazil), in: Associations volcano-plutoniques de l'Espinhaço Meridional (SE-Brésil). Tese de doutoramento Université d'Orleans, pp.23-52, Ined.

FERNANDES, M.L.S.; MARCIANO,V.R.P.R.O.; OLIVEIRA, R.C.de; DILÁSCIO, M.V. \& CORREIA NEVES, J.M. 1994. Petrografia e geoquímica de rochas granito-gnáissicas da região entre Itabira e Dores de Guanhães, MG. In: CONGR. BRAS. GEOL., 38. Camboriu, 1994. Bol. de Resumos Expandidos... pp.176-177.

GROSSI SAD, J. H.; CHIODI FILHO, C.; SANTOS, J. F.; MAGALHÃES, J. M. M. \& CARELOS, P. 1990 . Duas suítes graníticas do bordo sudeste do cráton Sanfranciscano, em Minas Gerais: petroquímica e potencial metalogenético. In: Anais do $36^{\circ}$ CONG. BRAS. GEOL., 36. Anais... 4:1836-1848.

HERZ,N. - 1970 - Gneissic and igneous rocks of the Quadrilátero Ferrífero, Minas Gerais, Brazil - US Geol. Surv., Prof. Pap. 641B, 58 p., Washington.

IRVINE,T.N.\& BARAGAR,W.R.A. 1971 . A guide to the chemical classification of the common volcanic rocks. Canadian J.Earth Sci, 8:523-548.

LA ROCHE,H. de; LETERRIER,J.; GRANDELANDE,P. \& MARSHSALL,M. 1980 . A classification of volcanic and plutonic rocks using R1R2 diagrams and major elements analysis: Its relationships with current nomenclature. Chem. Geol.,29:183-210.

MANIAR,P.D. \& PICCOLI,P.M. 1989. Tectonic discrimination of granitoids. Geol. Soc. Amer. Bull, 101:635-643.

MÜLLER,G. \& SCHORSCHER,J.H.D. 1977 . Untersuchungen zur Bildung metasomatischer Granitoide an einer tiefkrustalen Aufschiebung im präkambrischen Raum von Itabira, Minas Gerais, Brasilien. 1 Arbeitsbericht $\left(1^{\circ}\right.$ Relatório Científico).

PEARCE,J.A.; HARRIS,N.B.W. \& TINDLE,A.G. 1984 . Trace element discriminations diagrams for the tectonic interpretation of granitic rocks. J. Petrol. 25(4):956-983.

SCHORSCHER,J.H.D . 1992 . Granitóides Borrachudos e magmatismo granítico arqueano tardio, in: Arcabouço Petrográfico e Evolução Crustal de terrenos pré-cambrianos do Sudeste de Minas Gerais: Quadrilátero Ferrífero, Espinhaço Meridional e domínios granito-gnáissicos adjacentes. Tese de Livre Docência, 1:71-117, USP, Ined..

WHALEN,J.B.; CURRIE,K.L. \& CHAPPEL,B. 1987 . A-type granites: geochemical characteristics, discrimination and petrogenesis. Contr. Mineral Petrol., 95:407-419.

WRIGHT,J.B. 1969. A simple alkalinity ratio and its application to questions of non-orogenic granite genesis. Geol. Mag., 106(4):370-384

ZHONGGANG,W. 1982 . The REE distribution patterns of granitoids. - Procceedings of the International Symposium on Geology of Granites and their metallogenetic relations. Nanjing, China: 787-796. 
